\title{
Outcomes of patients with advanced non-small cell lung cancer and airway obstruction treated with photodynamic therapy and non-photodynamic therapy ablation modalities
}

\author{
Ravishankar Jayadevappa $^{1,2,3,4,5}$, Sumedha Chhatre $^{6}$, Harmik J. Soukiasian ${ }^{7}$, Septimiu Murgu $^{8}$ \\ ${ }^{1}$ Department of Medicine, ${ }^{2}$ Leonard Davis Institute of Health Economics, University of Pennsylvania, Philadelphia, PA, USA; ${ }^{3}$ Division of Urology, \\ Department of Surgery, ${ }^{4}$ Abramson Cancer Center, University of Pennsylvania School of Medicine, Philadelphia, PA, USA; ${ }^{5}$ Corporal Michael \\ J. Crescenz VAMC, Philadelphia, PA, USA; ${ }^{6}$ Department of Psychiatry, University of Pennsylvania School of Medicine, Philadelphia, PA, USA; \\ ${ }^{7}$ Cedars-Sinai Medical Center, Los Angeles, CA, USA; ${ }^{8}$ University of Chicago Medicine, Chicago, IL, USA \\ Contributions: (I) Conception and design: R Jayadevappa, S Chhatre; (II) Administrative support: R Jayadevappa; (III) Provision of study materials \\ or patients: R Jayadevappa, S Chhatre; (IV) Collection and assembly of data: R Jayadevappa, S Chhatre; (V) Data analysis and interpretation: R \\ Jayadevappa, S Chhatre; (VI) Manuscript writing: All authors; (VII) Final approval of manuscript: All authors. \\ Correspondence to: Ravishankar Jayadevappa, PhD. Department of Medicine, University of Pennsylvania, 3615 Chestnut St., R, 224, Philadelphia, PA \\ 19104, Email: jravi@pennmedicine.upenn.edu.
}

Background: Non-small cell lung cancer (NSCLC) patients with central airway obstruction (CAO) may have better survival on systemic therapy if the airway patency is successfully restored by bronchoscopic interventions. It remains unclear which therapeutic bronchoscopic modality [laser, stenting, external beam radiation, brachytherapy and photodynamic therapy (PDT)] used for restoring airway patency positively affects outcomes in these patients. We analyzed the effectiveness of PDT in terms of mortality, and time to subsequent treatments in patients with stage III and IV NSCLC.

Methods: Study used Surveillance, Epidemiology, and End Results (SEER) Medicare linked data. We categorized NSCLC patients diagnosed between 2000 and 2011 and with stage III and IV, into three treatment groups: PDT + radiation \pm chemotherapy, non-PDT ablation therapy + radiation \pm chemotherapy, and radiation + chemotherapy. We analyzed all-cause and cause-specific mortality using Cox proportional hazard models with an inverse probability weighted propensity score adjustment. Time to subsequent treatment was analyzed using GLM model.

Results: For the PDT group, hazard for all-cause and cause-specific mortality was comparable to the radiation + chemotherapy group $(\mathrm{HR}=1.03$, 95\% CI: $0.73-1.45$; and HR =1.04, 95\% CI: 0.71-1.51, respectively). The non-PDT ablation group had higher hazard for all-cause (HR =1.22, 95\% CI: 1.13-1.33) and cause-specific mortality ( $\mathrm{HR}=1.10,95 \% \mathrm{CI}: 1.01-1.20)$, compared to the radiation + chemotherapy group. The PDT group had longer time to follow-up treatment, compared to non-PDT ablation group.

Conclusions: In our exploratory study of stage III and IV NSCLC patients with CAO, addition of PDT demonstrated hazard of mortality comparable to radiation + chemotherapy group. However, addition of non-PDT ablation showed higher mortality compared to the radiation + chemotherapy group. Future studies should investigate the efficacy and effectiveness of multimodal therapy including radiation, chemo, immunotherapy and bronchoscopic interventions.

Keywords: Non-small cell lung cancer (NSCLC); photodynamic therapy (PDT); advanced stage; mortality

Submitted Jan 31, 2019. Accepted for publication Apr 11, 2019.

doi: $10.21037 /$ jtd.2019.04.60

View this article at: http://dx.doi.org/10.21037/jtd.2019.04.60

(C) Journal of Thoracic Disease. All rights reserved. 


\section{Introduction}

Lung cancer remains the leading cause of cancer death among men and women in the US. It is estimated that in year 2019, there will be about 228,150 new cases of lung cancer and approximately 142,670 deaths attributable to lung cancer (1). The Surveillance, Epidemiology, and End Results (SEER) data show that 5-year survival rate for lung cancer is $18.1 \%$ (2). Concurrent or sequential chemotherapy and external beam radiation therapy (RT) are standard of practice for patients with locally advanced non-small cell lung cancer (NSCLC) (3). For patients with loco-regional recurrence or symptomatic local disease with endobronchial obstruction, the 2019 NCCN lung cancer guidelines recommend any combination of the following: laser/stenting, RT/brachytherapy and photodynamic therapy (PDT) (3).

When lung cancer causes severe airway obstruction resulting in atelectasis, however, the response rate to RT alone is only about $20 \%(4,5)$, especially if the treatment is initiated two weeks or later after the development of atelectasis (6). In these patients with endobronchial obstruction undergoing RT, full restoration of airway patency could prolong the time interval from treatment to death by more than 4 months compared with those patients in whom recanalization failed (7). In fact, in a prospective study, the median survival in patients with malignant central airway obstruction (CAO) who underwent successful bronchoscopic intervention was better than in patients with unsuccessful intervention (229 vs. 115 days) (8). Therefore, in patients with locally advanced (stage III) or metastatic lung cancer (stage IV) and CAO, the published evidence and the ACCP lung cancer guidelines recommend therapeutic bronchoscopy to improve dyspnea, cough, hemoptysis and quality of life (8-12).

The modality used to restore airway patency (i.e., laser, stenting, PDT, electrocautery, or brachytherapy) depends on tumor characteristics (extrinsic, endoluminal, mixed), severity of the obstruction (critical, compromising respiratory status, stable), operator preference, bias, availability and expertise. PDT is a unique biopharmaceutical modality where a drug is selectively retained in tumors and activated by specific light allowing for destruction of tumor sites. PDT and other ablation therapies such as cryotherapy, argon plasma coagulation, and Nd:YAG laser have been effectively applied as monotherapy or multimodal approach for palliative care in NSCLC patients who are not surgical candidates (13). At least two studies suggest that there is no significant difference in the survival of patients with and without CAO from NSCLC, when the $\mathrm{CAO}$ is treated with ablative therapies, stenting or a combination in addition to systemic chemotherapy and radiation $(10,11)$. But it is known, however, that survival rate is significantly higher in patients with inoperable NSCLC without $\mathrm{CAO}$ than in patients with $\mathrm{CAO}(\mathrm{P}<0.001)(11)$. Because of these aforementioned reasons, clinicians routinely offer bronchoscopic interventions in patients with locally advanced or metastatic lung cancer and symptomatic $\mathrm{CAO}$ while continuing systemic oncologic treatment with chemotherapy and RT.

To date, the published literature on therapeutic bronchoscopy does not clarify whether the actual bronchoscopic modality used for restoring airway patency makes a difference in terms of outcomes. Currently, PDT may be underutilized due to several factors including perceived complexity of treatment given the requirement of a procedure (i.e., bronchoscopy) and administration of an oncologic drug (i.e., Photofrin); and lack of randomized controlled trials (RCT) comparing PDT and conventional therapies (e.g., thermal ablation, cryotherapy). The objective of this exploratory study was to assess the effectiveness of PDT in terms of mortality (all-cause and cause-specific), and time to subsequent treatments in fee-for service, Medicare patients with stage III or IV NSCLC. We analyzed patients who received PDT ablation or non-PDT ablation for endobronchial obstruction in addition to their systemic therapy and compared them with patients who only received RT and chemotherapy as part of their oncologic care.

\section{Methods}

\section{Data sources and cobort definition}

SEER-Medicare data from National Cancer Institute (NCI) link the Medicare administrative claims data and clinical tumor registry data for Medicare enrollees residing in the SEER regions (14). The SEER program encompasses $26 \%$ of the population of the US and gathers information regarding cancer incidence, treatment, and mortality from 20 SEER sites. Among cancer patients who are 65 years or older and are part of the SEER registries, $93 \%$ have been matched with Medicare enrollment records. In this retrospective cohort study, we used SEER-Medicare data to extract a cohort of patients aged 18 years or older, and diagnosed with stage III or IV NSCLC between 2000 and 2011. Local institutional review board at the University of Pennsylvania approved the study. 


\section{Measurement strategy}

\section{Key dependent variables under study}

Key dependent variables under study were mortality (allcause and cause-specific), and time to subsequent treatments (for PDT and non-PDT ablation groups only). Data on all-cause and cause-specific mortality were obtained using SEER's Patient Entitlement and Diagnosis Summary File (PEDSF). Time to death was defined as number of days between date of treatment and date of death. Additionally, those who survived up to the end of the study (12/31/2013) were censored. Time to subsequent treatment for PDT group and non-PDT ablation group was the time between first claim for PDT or non-PDT ablation therapy and subsequent claim for surgery or RT.

\section{Treatment type}

We reviewed inpatient, outpatient and provider claims to identify NSCLC treatments using International Classification of Disease (ICD)-9 and Healthcare Common Procedure Coding System (HCPCS) codes. We identified PDT + RT \pm chemotherapy, non-PDT ablation (argon plasma coagulation, cryotherapy, laser) + $\mathrm{RT} \pm$ chemotherapy, and RT + chemotherapy as the three exclusive treatment groups.

\section{Covariates}

Sociodemographic characteristics including age, race and ethnicity, gender, marital status, and geographic location were obtained from SEER's PEDSF database. Charlson comorbidity index score based on inpatient, outpatient and provider claims from the year prior to NSCLC diagnosis was calculated to measure medical comorbidities $(15,16)$.

\section{Analytic strategy}

First, we compared the demographic and clinical characteristics of NSCLC patients from the three different treatment groups: PDT $+\mathrm{RT} \pm$ chemotherapy, nonPDT ablation therapy + RT \pm chemotherapy, and RT + chemotherapy, using standard t tests and Chi-square tests. We used Cox regression model to determine the association between treatment group and mortality (all-cause and lung cancer-specific), after adjusting for covariates. We used GLM regression model to analyze the association between group membership (PDT $v s$. non-PDT ablation) and time to subsequent treatment, after adjusting for covariates. While assessing the relationship between treatment and outcomes among NSCLC patients, it is important to note that treatment assignment is non-random. Therefore, in order to minimize the observed bias due to treatment, we used propensity score approach. We used a two-stage propensity score technique for controlling the observed confounders. First, for each patient, we developed the probability or propensity of receiving $\mathrm{PDT}+\mathrm{RT} \pm$ chemotherapy, non-PDT ablation $+\mathrm{RT} \pm$ chemotherapy or RT + chemotherapy, as a function of age, gender, race and ethnicity, marital status, geographic location, cancer stage, and Charlson comorbidity score using multinomial logistic regression $(17,18)$. We then modeled the associations between treatment and mortality, and weighted it by the inverse probability of treatment obtained from the propensity score. To examine the degree of matching, we compared the distribution for the covariates pre and post adjustment for propensity score.

\section{Matched cohort analysis}

We performed a matched cohort analysis, with the PDT group as the focal group. For our PDT group, we extracted a 1:2 match from the non-PDT ablation group and the RT + chemotherapy group. The matching was done by age ( \pm 3 years), race, gender and comorbidity score. We then performed survival analysis and time-to follow-up treatment analysis using these three matched groups. All analyses were performed with the help of Statistical Analysis System (SAS), Version 9.3 (SAS Institute, Cary, NC).

\section{Results}

\section{Sample characteristics}

Our cohort consisted of 43,783, fee-for-service Medicare enrollees, older than 18 years, and diagnosed with stage III or IV NSCLC between 2000 and 2011 (see Figure 1 for cohort selection process). Of this cohort, 39 patients received $\mathrm{PDT}+\mathrm{RT} \pm$ chemotherapy, 558 received non$\mathrm{PDT}$ ablation $+\mathrm{RT} \pm$ chemotherapy and 43,186 received RT + chemotherapy. The details of the ICD codes and HCPCS codes used to identify our NSCLC cohort and the treatments are presented in Table S1. In Table 1, we present the demographic and clinical characteristics of our cohort. The average age of the patients from the three treatment groups was comparable. Compared to other groups, higher proportion of PDT group were Caucasian and male. The PDT group also had highest proportion of those with at least one medical comorbidity at the time of diagnosis of NSCLC (62\%), whereas this proportion was the lowest for 


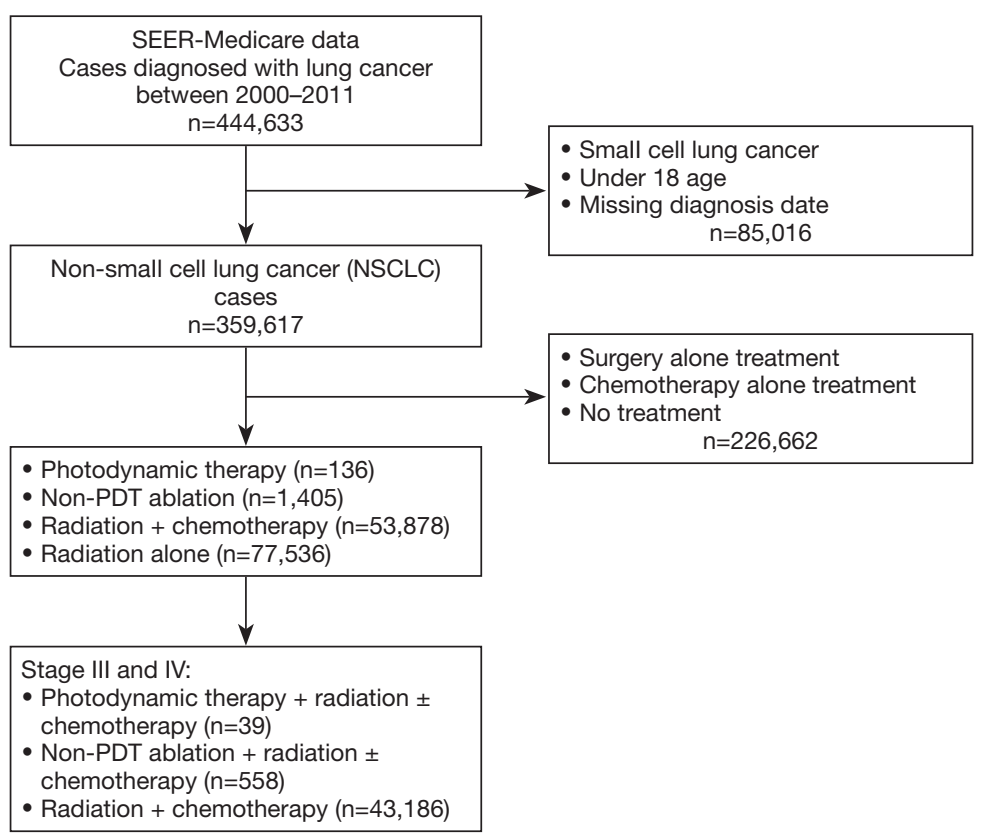

Figure 1 Flow chart for cohort selection.

Table 1 Comparison of baseline characteristics before and after propensity score weighting

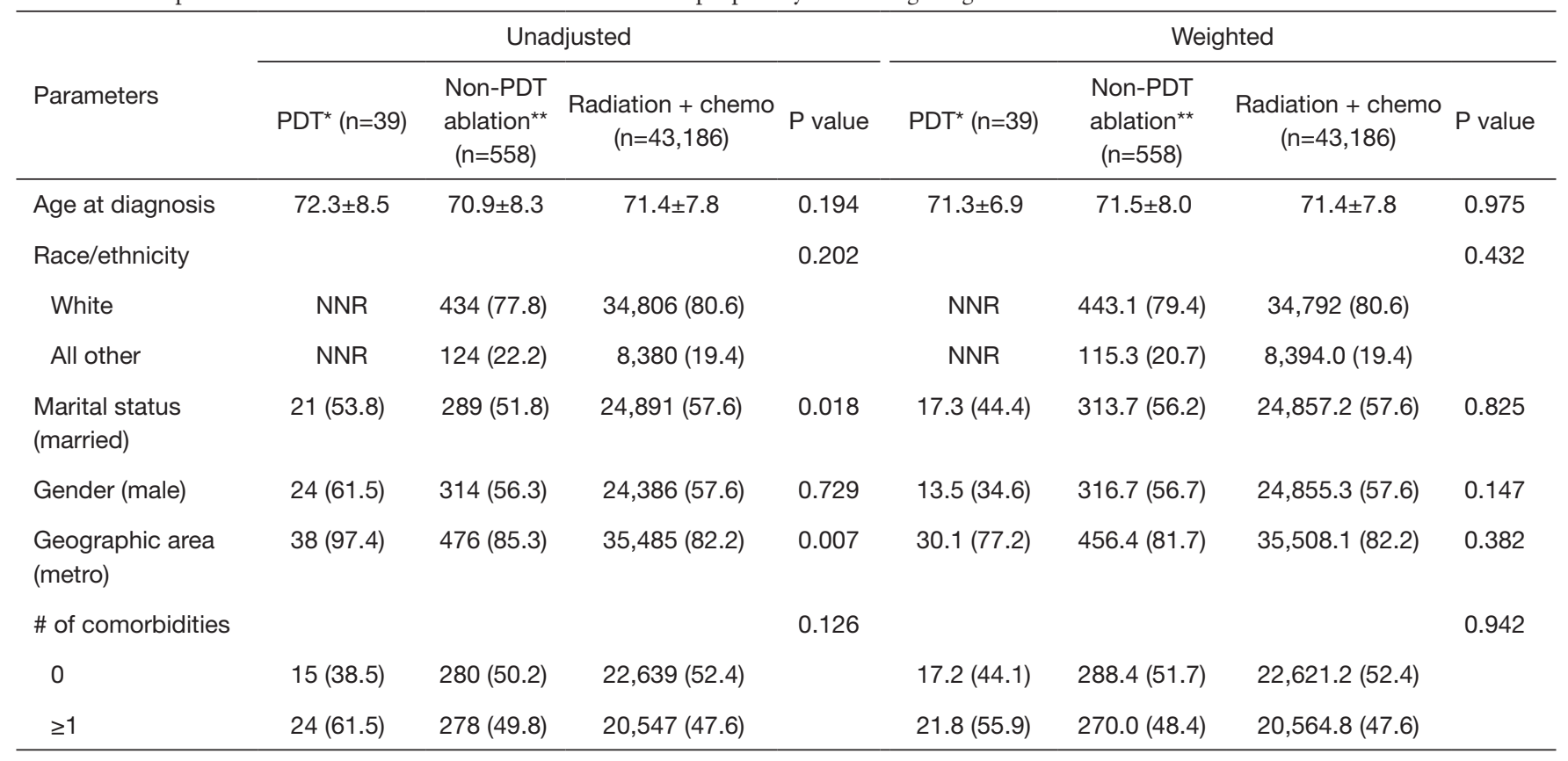

Data are shown as number (percentage) or mean \pm SD. *, PDT group includes PDT + radiation \pm chemotherapy; ${ }^{* *}$, non-PDT ablation group includes non-PDT ablation + radiation \pm chemotherapy. PDT, photodynamic therapy. NNR, numbers not reported due to small cell size. 
RT + chemotherapy group (48\%), however, this difference was not statistically significant. The propensity scores weighted comparison of demographic and clinical variables across the three treatment groups showed comparable distributions (Table 1).

Next, we computed the unadjusted survival time for the three treatment groups. The PDT group had the longest all-cause survival time [mean 505 days, standard deviation (SD) 526 days], followed by the RT + chemotherapy group (mean 502 days, SD 582 days), and non-PDT ablation group (mean 417 days, SD 497 days). Additionally, unadjusted comparison showed that compared to nonPDT ablation group, the PDT group had longer time to subsequent treatment (mean 147 days, SD 295 vs. mean 98 days, SD 233 days). Though this difference was not statistically significant $(\mathrm{P}=0.2048)$, this suggests that $\mathrm{PDT}$ may result in a more sustained response for NSCLC patients with endobronchial obstruction.

\section{All-cause and cause-specific mortality}

Results of Cox regression model for all-cause mortality are presented in Table 2. Compared to the RT + chemotherapy group, the propensity score adjusted hazard of all-cause mortality was similar for the PDT group ( $\mathrm{HR}=1.03,95 \%$ CI: 0.73-1.45); and higher for the non-PDT ablation group (HR $=1.22$, 95\% CI: $1.13-1.33)$. Table 2 also shows the results of Cox regression model for lung cancer-specific mortality. The propensity score adjusted hazard of causespecific mortality for PDT group was comparable to that of the RT + chemotherapy group (HR $=1.04,95 \%$ CI: 0.71-1.51). However, the non-PDT ablation group had higher hazard of cause-specific mortality ( $\mathrm{HR}=1.10,95 \%$ CI: 1.01-1.20), compared to the RT + chemotherapy group.

\section{Time to subsequent treatment}

Results of regression for association between group membership (PDT ablation vs. non-PDT ablation) and time to subsequent treatment are shown in Table 3. The propensity score adjusted regression showed that PDT group was associated with 29.2 days increase in time to follow-up treatment on average, however this increase was not statistically significant $(\mathrm{P}=0.4458)$.

\section{Matched cohort analysis}

Results of the matched cohort analysis are presented in
Table S2 and Table S3. The association between treatment groups and outcomes of mortality (all-cause and causespecific), and time to follow-up treatment was similar to that observed in the main analysis. As seen from Table $S 2$, compared to the RT + chemotherapy group, the propensity score adjusted hazard of all-cause mortality was similar for the PDT group (HR =1.30, 95\% CI: 0.85-1.98); and higher for the non-PDT ablation group (HR $=1.92,95 \% \mathrm{CI}$ : 1.38-2.70). Table $S 2$ also shows the results of Cox regression model for lung cancer-specific mortality. The propensity score adjusted hazard of cause-specific mortality for PDT group, and non-PDT ablation group was comparable to that of the RT + chemotherapy group ( $\mathrm{HR}=1.34,95 \% \mathrm{CI}$ : 0.84-2.15); and HR $=1.35$; 95\% CI: 0.94-1.94, respectively), compared to the RT + chemotherapy group. Results of regression for association between group membership (PDT ablation $v s$. non-PDT ablation) and time to subsequent treatment are shown in Table S3. The propensity score adjusted regression showed that PDT group was associated with 79.0 days increase in time to follow-up treatment on average $(\mathrm{P}=0.077)$.

\section{Discussion}

For patients with locally advanced NSCLC, guidelines recommend concurrent or sequential chemotherapy and RT (3). However, for patients with locoregional recurrence or symptomatic local disease with endobronchial obstruction, the NCCN and ACCP lung cancer guidelines recommend bronchoscopic interventions to improve dyspnea, cough, hemoptysis and quality of life $(3,12)$. These may include one or more of the following: laser, stenting, RT, brachytherapy and PDT. In fact, when lung cancer causes severe airway obstruction resulting in atelectasis, however, only about 1 in 5 patients will respond to RT alone (4-6). Survival in these patients is improved if the airway is opened successfully via bronchoscopic procedures. In a prospective study of 75 patients, RT and endobronchial laser resection were used to treat inoperable or recurrent bronchial carcinoma occluding a major airway. Complete recanalization was achieved in $36 \%$, partial recanalization in $51 \%$, and no recanalization in $13 \%$. These 75 patients were matched retrospectively with a group of 75 patients who received external RT for the same indications but no laser resection. Successful laser reopening of a major airway influenced the pattern of failure: with full recanalization respiratory failure was the cause of death in $23.3 \%$ of cases was whereas with 
Table 2 Association between treatment type and mortality

\begin{tabular}{|c|c|c|c|c|}
\hline Covariates & \multicolumn{2}{|c|}{ All-cause mortality } & \multicolumn{2}{|c|}{ Lung cancer-specific mortality } \\
\hline \multicolumn{5}{|l|}{ Treatment group } \\
\hline PDT $^{*}$ & 1.03 & $0.73-1.45$ & 1.04 & $0.71-1.51$ \\
\hline Non-PDT ablation ${ }^{\star \star}$ & 1.22 & $1.13-1.33$ & 1.10 & $1.01-1.20$ \\
\hline Age at diagnosis & 1.01 & $1.01-1.04$ & 1.00 & $1.00-1.01$ \\
\hline \multicolumn{5}{|l|}{ Race and ethnicity } \\
\hline White & 1.13 & $1.09-1.15$ & 1.04 & $1.01-1.07$ \\
\hline Other (reference) & - & - & - & - \\
\hline Other (reference) & - & - & - & - \\
\hline \multicolumn{5}{|l|}{ Gender } \\
\hline Male & 1.28 & $1.26-1.31$ & 1.03 & $1.01-1.05$ \\
\hline Female (reference) & - & - & - & - \\
\hline \multicolumn{5}{|l|}{ Geographic region } \\
\hline Metro & 0.94 & $0.92-0.97$ & 0.99 & $0.97-1.02$ \\
\hline Non-metro (reference) & - & - & - & - \\
\hline \multicolumn{5}{|l|}{ Commodity score } \\
\hline
\end{tabular}

*, PDT group includes PDT + radiation \pm chemotherapy; ${ }^{*}$, non-PDT ablation group includes non-PDT ablation + radiation \pm chemotherapy. PDT, photodynamic therapy.

no recanalization it was $56.3 \%$ (7). While laser resection did not influence the overall survival, in patients with full restoration of bronchial patency, the time interval from treatment to death was prolonged by more than 4 months compared with those patients in whom recanalization failed (7). In a more recent prospective study, the median survival in patients with malignant $\mathrm{CAO}$ who underwent successful bronchoscopic intervention was 229 days compared to 115 days in patients with unsuccessful intervention (8).

Evidence suggest that survival rate is significantly higher in patients with inoperable NSCLC without CAO than in patients with $\mathrm{CAO}(\mathrm{P}<0.001)(11)$. In addition, when the $\mathrm{CAO}$ is treated with ablative therapies, stenting or a combination in addition to systemic chemotherapy and RT, there is no significant difference in the survival of patients with and without CAO from NSCLC (10). Because of these aforementioned reasons, clinicians routinely offer palliative bronchoscopic interventions in patients with lung cancer and symptomatic $\mathrm{CAO}$ while continuing systemic oncologic treatment with chemotherapy and RT.

Our exploratory analysis of SEER-Medicare linked data shows that when compared to the RT + chemotherapy group, the propensity score adjusted hazard of all- 
Table 3 Association between treatment type and time to follow-up treatment

\begin{tabular}{|c|c|c|}
\hline Covariates & Parameter estimate & $P$ value \\
\hline Intercept & 302.0 & 0.001 \\
\hline \multicolumn{3}{|l|}{ Treatment group } \\
\hline $\mathrm{PDT}^{*}$ & 29.2 & 0.446 \\
\hline Non-PDT ablation** (reference) & - & - \\
\hline Age at diagnosis & -3.2 & 0.007 \\
\hline \multicolumn{3}{|l|}{ Race and ethnicity } \\
\hline White & 36.6 & 0.119 \\
\hline Other (reference) & - & - \\
\hline \multicolumn{3}{|l|}{ Marital status } \\
\hline Married & -57.9 & 0.005 \\
\hline Other (reference) & - & - \\
\hline \multicolumn{3}{|l|}{ Gender } \\
\hline Male & -51.7 & 0.012 \\
\hline Female (reference) & - & - \\
\hline \multicolumn{3}{|l|}{ Geographic region } \\
\hline Metro & 29.1 & 0.307 \\
\hline Non-metro (reference) & - & - \\
\hline \multicolumn{3}{|l|}{ Commodity score } \\
\hline Comorbidity $\geq 1$ & -14.3 & 0.456 \\
\hline Zero comorbidity (reference) & - & - \\
\hline \multicolumn{3}{|l|}{ Stage } \\
\hline Stage III & 56.3 & 0.004 \\
\hline Stage IV (reference) & - & - \\
\hline
\end{tabular}

cause mortality was similar for the PDT group. In other words, the risk of death in patients with endobronchial obstruction treated with PDT and RT \pm chemotherapy is similar to those without $\mathrm{CAO}$ who were treated with $\mathrm{RT} \pm$ chemotherapy. This is consistent with the findings from the studies by Chhajed et al. and Chen et al. $(10,11)$. Chhajed et al. (10) studied two groups. In group A ( $\mathrm{n}=52)$ CAO from NSCLC was treated with: laser alone in 13 patients $(25 \%)$, stent insertion alone in 13 patients $(25 \%)$, both stent + laser in $26(50 \%)$. Chemo +/- RT was used in 33 patients (63\%). In Group B ( $\mathrm{n}=92)$, there was no CAO, and these patients were treated with systemic therapy alone. Median survival in patients belonging to group $\mathrm{A}$ was 8.4 months and those in group B was 8.2 months and there was no significant difference in the survival of patients with and without $\mathrm{CAO}$ $(\mathrm{P}=0.395)$. Similarly, Chen et al. (11) showed that survival rate was significantly higher in patients with inoperable lung cancer but no CAO than in patients with $\mathrm{CAO}(\mathrm{P}<0.001)$. However, there was no statistically significant difference in the survival between patients with inoperable lung cancer and CAO after therapeutic bronchoscopy and patients without $\mathrm{CAO}(\mathrm{P}=0.101)$. Our study suggests that patients with lung cancer and $\mathrm{CAO}$ treated with PDT and RT \pm chemotherapy have similar death risk as patients without $\mathrm{CAO}$ treated with $\mathrm{RT} \pm$ chemotherapy alone. This is extremely relevant for clinicians as restoring airway patency with bronchoscopic interventions could be risky in an already debilitated patient population. As discussed above, however, just continuing RT for CAO is only successful in a minority of patients and is no surprise that restoring airway patency in these patients could improve outcomes. Also, when compared with the RT + chemotherapy group, the comparable hazards of mortality in the PDT group $v s$. higher hazards of mortality in the non-PDT ablation group may suggest that PDT is advantageous than nonPDT ablation in adjusting the patient back to the nonCAO state. This phenomenon is likely explained by PDT's ability to treat unseen tumor margins. In fact, studies in animal models have proven that untreated tumor margins are responsible for early (within a few weeks) recurrence, a phenomenon that can be potentially prevented by the use of PDT, which treats visible and non-visible tumor (19).

Additionally, we found that PDT ablation group had a trend towards longer time to subsequent treatment compared to non-PDT ablation therapy, suggesting that PDT has a more durable anti-cancer effect. This observation may be due to the fact that PDT achieves selective necrosis of up to $6 \mathrm{~mm}$ depth and treats visible and nonvisible disease, contrary to thermal [laser, contact electrocautery and non-contact electrocautery (i.e., argon plasma coagulation)] or cryotherapy ablative treatments that address only visible disease areas.

In addition, we found that despite similar comorbidity scores, the non-PDT ablation group had higher hazard of all cause and cause-specific mortality compared to the RT + chemotherapy group. This is probably because of the undesired consequences of $\mathrm{CAO}$, which was presumably not present in the chemo + RT group. The explanation for better outcomes with PDT ablation remains speculative but 
may be due to a more sustained local control, safety profile or due to abscopal effect through PDT-induced immunogenic properties, similar to that described for RT (20). Research indicates that local PDT treatment can lead to systemic neutrophilia, induction of acute phase reactants, increased circulating levels of complement levels, and systemic release of inflammatory cytokines (21). Together, these are indicative of systemic inflammatory response with T-cell mediated anti-tumor immune response. Studies have also shown that local PDT treatment of murine tumors can result in the induction of anti-tumor immunity, which may control distant, untreated disease sites (21). In human studies, there are reports of increased survival for patients with NSCLC with malignant pleural disease treated with surgery and PDT, compared to those treated with surgery alone (i.e., historical controls) (22). These results indicate that local PDT treatment of tumors may initiate an anti-tumor immune response that is able to control the growth of tumors external to the treatment field. Thus, this modality can be potentially useful for treating distant disease sites $(23,24)$. Results of preclinical and clinical studies indicate that along with its direct effects on tumor cell, PDT also boosts antitumor immunity and improves tumor cell immunogenicity. Further trials evaluating PDT should monitor immune markers prior to and after the PDT treatment.

Published data suggests that improvement in survival can be seen with interventional bronchoscopy and use of a variety of endobronchial treatment modalities for airway obstruction when compared with a single modality treatment. In one study, a single-modality treatment group included Nd:YAG laser, stenting, brachytherapy, and PDT. A variety of combinations of the aforementioned modalities were used in the other group to improve airway patency. There was a significant improvement in survival for the multimodality group $(\mathrm{P}=0.04)$. The 1 - and 3 -year cumulative survival rate for the two groups were $51.3 \%$ vs. $50 \%$ and $2.3 \%$ vs. $22 \%$, respectively (favoring multimodal approach) (25). Our analysis did not address the role of multimodal bronchoscopic interventions for restoring airway patency. Future studies should compare these techniques in a prospective fashion. Indeed, only two prospective RCTs of PDT compared to Nd:YAG laser ablation among patients with partially obstructive lung cancer demonstrated the superiority of PDT compared to Nd:YAG laser for alleviating dyspnea, cough, and hemoptysis $(26,27)$. While all-cause, adverse reactions were comparable between PDT and Nd:YAG laser, it was observed that up to one-fifth of PDT group exhibited photosensitivity reaction, probably stemming from noncompliance with precaution measures (26,28-31). This reaction can be prevented with proper patient and family education. There are several reasons that have historically limited a broader implementation of bronchoscopic PDT among proceduralists. These include but are not limited to: (I) complex scheduling and need for multiple procedures in the days following light application. A follow-up bronchoscopy at 48 hours after illumination is mandatory for cleaning up the tumor debris and to restore airway patency, but in patients with severe obstruction, more than one procedure may be necessary, especially if repeat light application is performed; (II) higher initial cost due to Photofrin infusion, repeat bronchoscopies and inpatient observation post procedure when compared with the immediate relief therapies such as laser, APC and contact electrocautery; (III) potential negative impact on the quality of life due to photosensitivity precautions in the weeks following PDT.

We believe that with proper patient education offered on at least three occasions (at the initial clinic appointment, at the time of the Photofrin infusion and at the time of PDT light application), photosensitivity reactions are preventable. However, newer photosensitizers which can be administered immediately pre-procedure and with a shorter photosensitivity risk period are desirable but as of this writing, they are not yet commercially available in the US. More studies are needed to assess the costeffectiveness and the quality of life in patients undergoing palliative bronchoscopic interventions. In the only prospective studies comparing PDT with an immediate relief therapy (Nd:YAG laser), however, PDT was found superior for alleviating symptomatology $(26,27)$. In fact, in one study, the PDT effect was more durable and resulted in improved survival (27). Our SEER database analysis adds to the body of evidence that this may indeed be the case, but prospective randomized studies will be necessary for confirmation. In addition, it is likely that multimodal bronchoscopic interventions will become more common as our understanding of local tissue and systemic effects continues to improve. In these regards, future studies should compare the adjuvant role of PDT after immediate relief therapies with the hypothesis that the bed of the tumor may be treated with PDT which will probably ensure a more durable effect due to treatment of non-visible disease and PDT-related anti-tumor local and potential systemic immunity. 
We believe our study adds to the body of evidence that PDT should be incorporated in the management of patients with locally advanced or metastatic NSCLC and symptomatic CAO just as recommended by guidelines (3). PDT, however, should be seen as a modality complementary to RT and chemotherapy. In fact, in a prospective trial of PDT plus RT versus RT alone, only $10 \%$ of patients in the RT only group achieved complete opening of the obstructed airway. On the other hand, this proportion was $70 \%$ for the PDT plus RT group (32). Therefore, for patients with inoperable disease, PDT should be incorporated as part of multimodal approach in conjunction with RT and potentially with immuno- or conventional chemotherapy. This is reasonable as in patients with bronchial obstruction from NSCLC, PDT improves performance status scores in more than $80 \%$ of patients, which may allow initiation or continuation of systemic anti-cancer therapy (33). In fact, the use of PDT does not preclude additional or subsequent treatment options. RT, however, will need to be delayed for about 4 weeks after PDT to avoid a profound effect that may injure the airway wall and potentially lead to stenosis or fistulas. Newer photosensitizers, better bronchoscopic and imaging techniques and focused information dissemination can enhance the usefulness of PDT as an effective modality for NSCLC with CAO.

\section{Limitations}

Our study has some limitations. First, due to the observational nature of our data, we were not able to establish a causal relationship between PDT and the outcomes. Second, even after using propensity score to minimize bias, some residual bias may exist. Third, the SEER-Medicare database allows for propensity matching to adjust for differences in treatment types for each stage of disease but the presence or absence of intraluminal disease is not clear in the RT+ chemotherapy group. However, given the fact that patients with symptomatic CAO routinely receive some form of bronchoscopic intervention as recommended by guidelines, it is reasonable to assume that the RT + chemotherapy group did not have CAO. Our cohort consisted of fee-for-service Medicare beneficiaries aged 18 years and older, not enrolled in an HMO and living in a SEER region. The age and sex distribution for persons 66 years and older is comparable with that of older adults in the US, however, SEER regions have a higher proportion of non-white persons. Additionally, the mortality rates obtained from SEER data may not represent national cancer mortality rates (14).

Our study is also potentially limited by our use of the SEER database. While ambiguous data input may occur in some instances, this is the only feasible method for a large database like SEER. Validity of data may be questioned in regards to the assumption that patients who received chemoradiation alone did not have concurrent CAO, especially since in years 2000-2011, the IP training programs in US were still growing. The study could be impacted by these missing data regarding concurrent $\mathrm{CAO}$ in patients who received chemotherapy $+\mathrm{RT}$ alone. However, according to our analysis, patients in the chemotherapy + RT group did not receive any form of surgical intervention or bronchoscopic ablation as identified by the CPT code 31641 procedure ("relief of obstruction any method"). In addition, based on the standard of care for patients with CAO for several decades, it is unlikely that the chemotherapy + RT group patients in the present study had any significant symptomatic airway obstruction. Even if some of them did, when compared with the RT + chemotherapy group, the comparable hazards of mortality in the PDT group vs. higher hazards of mortality in the non-PDT ablation group suggests the benefit of PDT $v s$. non-PDT ablation. These findings, however, will need confirmation in larger studies as the Figure 1 illustrates, out of the 43,783 total patients with stage III and IV, in this analysis ablative bronchoscopic ablative interventions were performed in only 597 patients (1.36\%).

Despite of these limitations, ours is the first study using large administrative data to demonstrate that PDT as part of multimodal approach is a meaningful anti-cancer treatment modality for patients with stage III or IV NSCLC with airway obstruction with potential for improved outcomes when compared with non-PDT ablation techniques and a trend towards longer time to subsequent treatments. Racial and social disparities observed in this analysis deserve further investigations, especially as we found that PDT ablation usage was more common among patients who were Caucasian, and of male gender.

\section{Conclusions}

We found that in stage III or IV NSCLC patients with $\mathrm{CAO}$, addition of PDT demonstrated hazard of mortality comparable to the radiation + chemotherapy group. Our results add to the body of evidence regarding incremental value of PDT ablation strategies in patients with stage III and IV NSCLC with airway involvement. Currently, PDT 
is offered as a standalone modality for early airway lung cancer or as part of multimodal approach in combination with surgery, RT or chemotherapy. Future prospective studies should focus on the systemic immune effects of PDT, its role for treating inoperable peripheral lung cancer, and its potential role in combination with immunotherapy in patients with NSCLC.

\section{Acknowledgments}

This study used the linked SEER-Medicare database. We acknowledge the efforts of the Applied Research Program, National Cancer Institute (NCI); the Office of Research, Development and Information, Centers for Medicare and Medicaid Services (CMS); Information Management Services; and the SEER program tumor registries in the creation of the SEER-Medicare database. This work was supported by an educational grant from Pinnacle Biologics.

\section{Footnote}

Conflicts of Interest: Drs. Soukiasian and Murgu are Educational Consultants for Pinnacle Biologics. There are no financial/nonfinancial conflicts of interest for other authors.

Ethical Statement: Local institutional review board at the University of Pennsylvania approved the study.

Disclaimer: The interpretation and reporting of these data are the sole responsibility of the authors.

\section{References}

1. American cancer Society. Available online: https://www. cancer.org/content/dam/CRC/PDF/Public/8703.00.pdf

2. Ettinger DS, Akerley W, Bepler G, et al. Non-Small Cell Lung Cancer. J Natl Compr Canc Netw 2010;8:740-801.

3. NCCN Clinical Practice Guidelines in Oncology. Non Small Cell Lung Cancer. Available online: https://www. nccn.org/professionals/physician_gls/pdf/nscl.pdf

4. Chetty KG, Moran EM, Sassoon CS, et al. Effect of radiation therapy on bronchial obstruction due to bronchogenic carcinoma. Chest 1989;95:582-4.

5. Slawson RG, Scott RM. Radiation therapy in bronchogenic carcinoma. Radiology 1979;132:175-6.

6. Reddy SP, Marks JE. Total atelectasis of the lung secondary to malignant airway obstruction Response to radiation therapy. Am J Clin Oncol 1990;13:394-400.
7. Macha HN, Becker KO, Kemmer HP. Pattern of failure and survival in endobronchial laser resection. A matched pair study. Chest 1994;105:1668-72.

8. Mahmood K, Wahidi MM, Thomas S, et al. Therapeutic bronchoscopy improves spirometry, quality of life, and survival in central airway obstruction. Respiration 2015;89:404-13.

9. Ong P, Grosu HB, Debiane L, et al. Long-term qualityadjusted survival following therapeutic bronchoscopy for malignant central airway obstruction. Thorax 2019;74:141-56.

10. Chhajed PN, Baty F, Pless M, et al. Outcome of treated advanced non-small cell lung cancer with and without central airway obstruction. Chest 2006;130:1803-7.

11. Chen $\mathrm{CH}, \mathrm{Wu} \mathrm{BR}$, Cheng WC, et al. Interventional pulmonology for patients with central airway obstruction: An 8-year institutional experience. Medicine 2017;96:e5612.

12. Simoff MJ, Lally B, Slade MG, et al. Symptom management in patients with lung cancer: Diagnosis and management of lung cancer, 3rd ed: American College of Chest Physicians evidence-based clinical practice guidelines. Chest 2013;143:e455S-e497S.

13. Detterbeck FC, Lewis SZ, Diekemper R, et al. Execuative Summary-Diagnosis and Management of Lung Cancer, 3rd ed: American College of Chest Physicians Evidence-Based Clinical Practice Guidelines. Chest 2013;143:7S-37S.

14. Warren JL, Klabunder CN, Schrag D, et al. Overview of the SEER-Medicare data-content, research application and generalizability to the United States elderly population. Med Care 2002;40:IV-3-18.

15. Elixhauser A, Steiner C, Harris DR, et al. Comorbidity measures for use with administrative data. Med Care 1998;36:8-27.

16. Klabunde CN, Warren JL, Legler JM. Assessing comorbidity using claims data-an overview. Med Care 2002;40:IV-26-35.

17. Green WH. Econometric analysis. Upper Saddle River, New Jersey: Prentice Hall; 2000.

18. Spreeuwenberg MD, Bartak A, Croon MA, et al. The multiple propensity score a control for bias in the comparison of more than two treatment arms-an introduction from a case study in mental health. Medical Care 2010;48:166-74.

19. Shafirstein G, Bellnier DA, Oakley E, et al. Irradiance controls photodynamic efficacy and tissue heating in experimental tumours: implication for interstitial PDT of 
locally advanced cancer. Br J Cancer 2018;119:1191-9.

20. Reynders K, Illidge T, Siva S, et al. The abscopal effect of local radiotherapy: using immunotherapy to make a rare event clinically relevant. Cancer Treat Rev 2015;41:503-10.

21. Gollnick SO, Brackett CM. Enhancement of anti-tumor immunity by photodynamic therapy. Immunol Res 2010;46:216-26.

22. Friedberg JS, Mick R, Stevenson JP, et al. Phase II trial of pleural photodynamic therapy and surgery for patients with non-small-cell lung cancer with pleural spread. J Clin Oncol 2004;22:2192-201.

23. Kabingu E, Vaughan L, Owczarczak B, et al. CD8+ T cell-mediated control of distant tumours following local photodynamic therapy is independent of CD4+ T cells and dependent on natural killer cells. Br J Cancer 2007;96:1839-48.

24. Pizova K, Tomankova K, Daskova A, et al. Photodynamic Therapy for engancing antitumor immunity. Biomed Pap Med Fac Univ Palacky Olomouc Czech Repub 2012;156:93-102.

25. Santos RS, Raftopoulos Y, Keenan RJ, et al. Bronchoscopic palliation of primary lung cancer: single or multimodality therapy? . Surg Endosc 2004;18:931-6.

26. Usuda J, Kato H, Okunaka T, et al. Photodynamic Therapy (PDT) for Lung Cancers. J Thorac Oncol

Cite this article as: Jayadevappa R, Chhatre S, Soukiasian HJ, Murgu S. Outcomes of patients with advanced non-small cell lung cancer and airway obstruction treated with photodynamic therapy and non-photodynamic therapy ablation modalities. J Thorac Dis 2019;11(10):4389-4399. doi: 10.21037/jtd.2019.04.60
2006;1:489-93.

27. Diaz-Jiménez JP, Martínez-Ballarín JE, Llunell A, et al. Efficacy and safety of photodynamic therapy versus $\mathrm{Nd}$ YAG laser resection in NSCLC with airway obstruction. Eur Respir J 1999;14:800-5.

28. Cortese DA, Edell ES, Kinsey JH. Photodynamic therapy for early stage squamous cell carcinoma of the lung. Mayo Clin Proc 1997;72:595-602.

29. Dolmans DE, Fukumura D, Jain RK. Photodynamic therapy for cancer. Nat Rev Cancer 2003;3:380-7.

30. Dougherty TJ, Gomer CJ, Henderson BW, et al. Photodynamic Therapy. J Natl Cancer Inst 1998;90:889-905.

31. Simone CB II, Friedberg JS, Glatstein E, et al. Photodynamic therapy for the treatment of non-small cell lung cancer. J Thorac Dis 2012;4:63-75.

32. Lam S, Crofton C, Cory P. Combined Photodynamic Therapy (PDT) using Photofrin and radiotherapy (XRT) versus radiotherapy alone in patients with inoperable distribution non-small cell bronchogenic cancer. Proc SPIE 1991:20-8.

33. Moghissi K, Dixon K, Stringer M, et al. The place of bronchoscopic photodynamic therapy in advanced unresectable lung cancer: experience of 100 cases. Eur J Cardiothorac Surg 1999;15:1-6. 